\title{
Peroxiredoxin-5 is a negative survival predictor in ovarian cancer
}

\author{
Jacek Sienko ${ }^{1}$, Pawel Gaj ${ }^{2}$, Krzysztof Czajkowski ${ }^{1}$, Dominika Nowis ${ }^{3,4,5}$ \\ ${ }^{1} 2^{\text {nd }}$ Department of Obstetrics and Gynecology, Medical University of Warsaw, Poland \\ ${ }^{2}$ Laboratory of Human Cancer Genetics, Center of New Technologies, University of Warsaw, Poland \\ ${ }^{3}$ Department of Immunology, Center for Biostructure Research, Medical University of Warsaw, Poland \\ ${ }^{4}$ Laboratory of Experimental Medicine, Center of New Technologies, University of Warsaw, Poland \\ ${ }^{5}$ Department of Genomic Medicine, Medical University of Warsaw, Poland
}

\begin{abstract}
Objectives: Peroxiredoxins (PRDXs) constitute a family of antioxidant enzymes which are also involved in the process of carcinogenesis. They are composed of six identified isoforms (PRDX-1-6) and are supposed to play different roles in tumor progression, depending on type of cancer and member of the PRDX family. The aim of the study was to assess the prognostic value of PRDXs in ovarian cancer.

Material and methods: a dataset of patients with ovarian cancer from The Cancer Genome Atlas was analyzed. Expression of PRDX-1 to 6 mRNA was evaluated in 260 samples. The prognostic value of PRDXs was assessed using the Cox regression model which included the following clinical and pathological data: age, clinical stage, tumor grade, and residual disease. Results: Within the PRDXs family, only higher expression of PRDX-5 was associated with worse overall survival both, in unselected patients and >50-year-olds. PRDX-5 expression and residual disease were independent negative prognostic factors of patient survival.
\end{abstract}

Conclusions: PRDX-5 is a negative predictor of survival in ovarian cancer.

Key words: peroxiredoxin-5; ovarian cancer; prognostic factor; overall survival

Ginekologia Polska 2019; 90, 1: 1-6

\section{INTRODUCTION}

Ovarian cancer $(\mathrm{OC})$ remains the leading cause of cancer-related deaths among females in the developed countries [1]. High mortality rate is the consequence of late diagnosis, which in turn is the result of relatively oligosymptomatic course of early-stage OC and lack of screening tests which could be used in low-risk populations [2]. Despite significant progress in the management of ovarian cancer, the overall 5-year survival for all stages of the disease is about 30\% [3]. Little is known about the predictive factors which would indicate a specific and more effective strategy of treatment. Cisplatin and Poly-(ADP-ribose)-polymerase1 (PARP1) administration in BRCA-mutated patients is one of the exceptions [4]. Resistance to chemotherapy is another major problem in a successful OC treatment. Overcoming primary or secondary resistance to chemotherapy requires a multidirectional strategy, which includes better knowledge of the biomarkers for survival prediction and are supposed to be involved in the development of resistance to chemotherapeutic agents.

Peroxiredoxins (PRDXs) are a ubiquitous family of antioxidant enzymes which also control cytokine-induced peroxide levels which mediate signal transduction in mammalian cells [5]. They are composed of six identified isoforms: PRDX1, PRDX2, PRDX3, PRDX4, PRDX5, and PRDX6 [6]. Under physiological conditions, they are responsible for protecting cells against oxidative DNA damage and genomic instability, regulating cell signaling associated with $\mathrm{H} 2 \mathrm{O} 2$, influencing cell differentiation and proliferation, immune responses and apoptosis [7]. In vitro and in vivo studies have demonstrated that the overexpression of PRDXs may either inhibit cancer development or promote cancer growth, depending on the specific PRDX family member and the cancer context [8]. PRDX-1 and PRDX-2 are the two repre- 
sentatives of the PRDX family which have a dual effect in carcinogenesis. PRDX-1 is overexpressed in lung, bladder and ovarian cancers, in aggressive esophageal squamous carcinomas, hilar cholangiocarcinoma, liver and pancreatic cancer, in mesothelioma and in glioblastoma, whereas high levels of PRDX-2 were observed in ovarian borderline tumors, colorectal cancers, vaginal, cervical, prostate and esophageal cancers and B-cell-derived primary lymphoma cells, suggesting their role in tumor progression [7]. However, biomarker studies demonstrated that PRDX-1 protects estrogen receptors (ERa) from the oxidative stress-induced suppression and is a protein marker of favorable prognosis in mammary tumors [9]. A decreased expression of PRDX-2 has been demonstrated in only a few types of cancer, including the melanomas, where the downregulation of PRDX-2 correlated with increased proliferative and migratory activities, and with the acquisition of a metastatic potential of the tumor [10]. The studies on PRDX-3 have demonstrated that its overexpression in hepatocellular, lung, breast, prostate and cervical cancers correlates with a more aggressive phenotype [11-15]. PRDX-4 enhances the rate of cell proliferation in prostate cancer and increases metastatic potential in oral cavity squamous cell, breast, lung and ovarian cancers [16-19]. Overexpression of PRDX-5 has been found in aggressive Hodgkin's lymphomas, malignant mesothelioma, breast, ovarian and thyroid cancer [20-24]. Increased levels of PRDX- 6 have been associated with a more invasive phenotype and metastatic potential of breast cancer, and with a worse prognosis of clinically localized prostate cancer following radical prostatectomy $[25,26]$. Finally, high levels of PRDX-1, 2, 3 and 6 correspond to resistance to cisplatin in several cancers [7]. The undisputable role of PRDXs in tumorigenicity makes these proteins candidates for markers of prognosis. Possibly, in the future research on such novel biomarkers may help identify patients who require specific, personalized approach, which could be another step towards improving the OC outcomes.

\section{Objectives}

The aim of our study was to investigate the prognostic significance of the PRDX family overexpression at the mRNA level in patients with ovarian cancer.

\section{MATERIAL AND METHODS}

The ovarian cancer normalized Agilent G4502A data (level 3) was acquired from The Cancer Genome Atlas (TCGA) (https://www.nature.com/articles/nature10166).TCGA is a collaboration between the National Cancer Institute $(\mathrm{NCl})$ and the National Human Genome Research Institute (NHGRI), which generated multi-dimensional maps of the key genomic changes in 33 types of cancer with poor prognosis. The dataset is publicly available and includes sam- ples from patients with ovarian cancers. In this study, we analyzed the expression of PRDX-1 to 6 mRNA in patients with ovarian cancer. A total of 270 subjects were included in the study. In order to run a multivariable Cox proportional hazard model for the overall survival, the following additional independent variables were included: age, clinical stage, tumor grade, and residual disease. A subgroup of 215 patients $>50$ years of age was selected from the study population to focus our study on the late-onset patients. The patients were further stratified into groups according to the quantiles of PRDX mRNA levels defining the following thresholds: Q1 - lower quartile, Q2 median, Q3 upper quartile. Characteristics of the entire study population and the group of $>50$-year-olds are presented in Tables 1 and 2 .

Kaplan-Meier plots were generated to visualize the differences in the overall survival between selected subgroups of the investigated OC patients. Differences in the survival were statistically compared using the Cox proportional hazard regression model to simultaneously assess the effect of additional risk factors on the survival. The $p$-value of $<0.05$ was considered as statistically significant. All analyses were performed using $R$. Local Ethics Committee approved of the study.

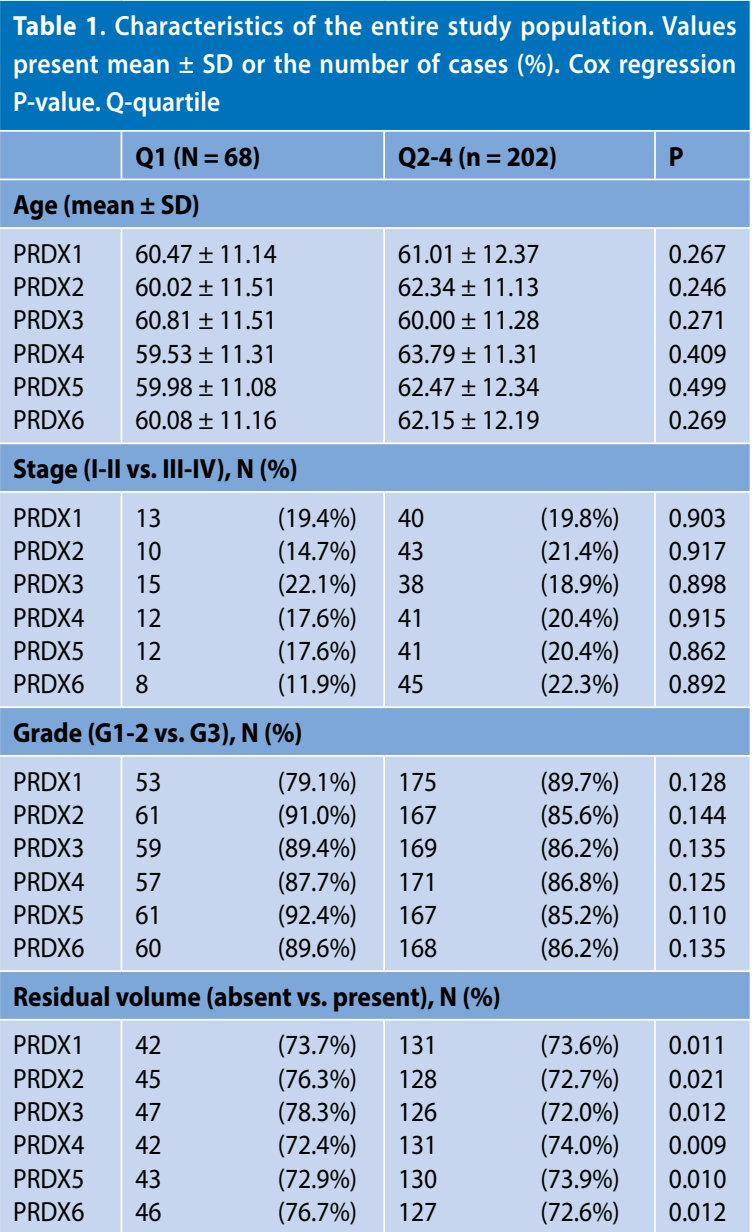




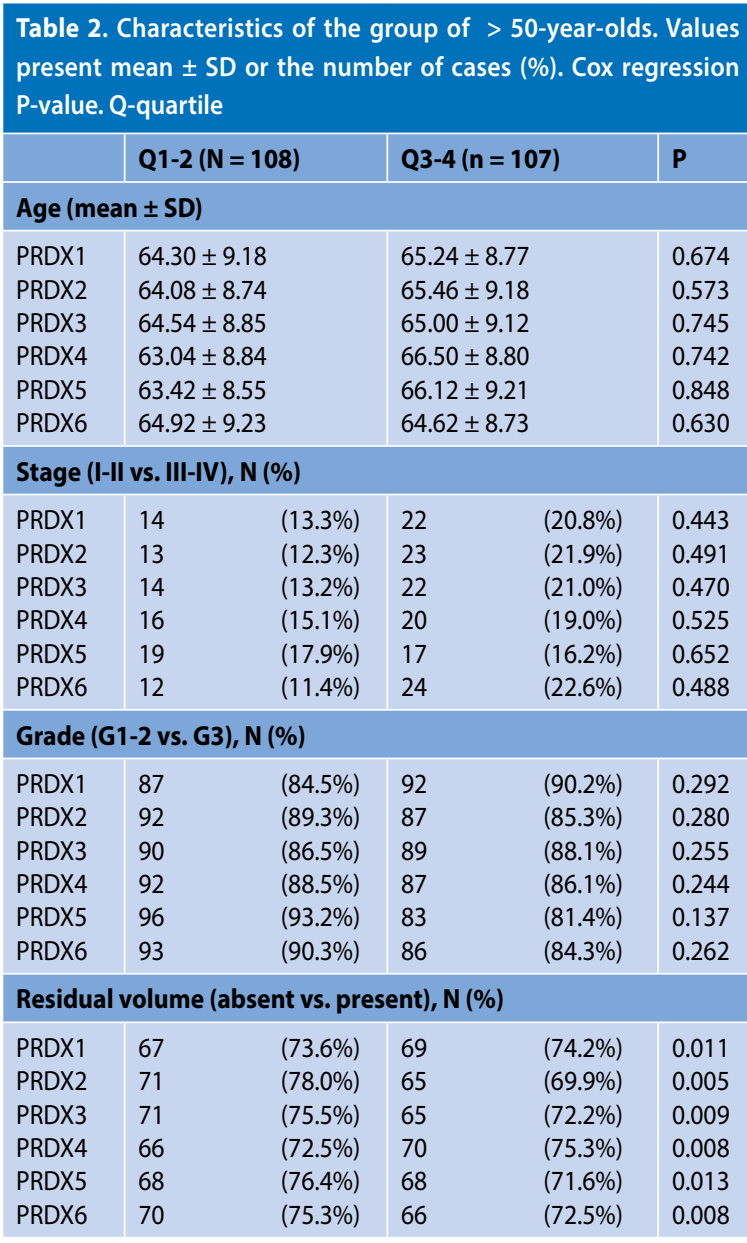

\section{RESULTS}

The overall survival multivariate analysis revealed that higher expression of PRDX-5 was independently associated with worse overall survival within the first five years after the initial diagnosis. The difference was observed in the entire study population for the Q1 threshold (within the $1^{\text {st }}$ quartile) $(P<0.05)$ (Fig. 1), and in the group of $>50$-year-olds for the Q2 threshold (within the median) $(P<0.04)$ (Fig. 2). Median overall survival was $1364(95 \% \mathrm{Cl} 1162-1652)$ days vs. 1919 (95\%Cl 1418-NA) days in the entire group and 1364 (95\%Cl 1184-1882) days vs. 1919 (95\%Cl 1684-2780) days in the group of $>50$-year-olds. No differences in the survival between the groups with different expression of PRDX-1, 2, 3, 4 and 6 were found. The results also revealed a significant influence of the residual disease on the reduced overall survival in the investigated cases $(P=0.01$ and $\mathrm{P}=0.013$, in all patients and $>50$-year-olds, respectively). In contrast, the clinical stage, tumor grade and age did not indicate significant effects on the overall survival.

\section{DISCUSSION}

PRDXs play a critical role in several physiological and pathological conditions involving redox signaling. Although their role in some benign conditions has been well understood, the influence of PRDXs on carcinogenesis remains controversial $[8,27]$. Different PRDX isoforms may have a tumorsuppressor or an oncogenic role, depending on the cancer type [7]. Thus, it is impossible to create a universal description of PRDXs as prognostic factors in mammalian malignant tumors. Regardless of the fact that several studies of PRDXs in various cancers have been published, little is known about their impact on the prognosis in OC patients. In the present study, we investigated the role of all six members of the PRDX family as markers of prognosis in ovarian cancer. According to our results, a higher expression of PRDX- 5 is associated with poorer overall survival both, in all ovarian cancer patients and in the group of $>50$-year-olds.

Our findings support the claims presented in other studies that PRDX-5 may give tumor-promoting effects in mammalian cancers. On the molecular level, transcription factors such as AP1, nuclear factorkB (NFKB), antioxidant response element, insulin response element, glucocorticoid response element, and also cMyc may directly modulate the expression of PRDX5 [28, 29]. CMyc also contributes to the maintenance of reactive oxygen species homeostasis through its ability to selectively induce the transcription of specific PRDXs when the function of one of them is compromised [29]. In clinical studies, the overexpression of PRDX-5 was significantly associated with tumor size and depth, and lymphatic invasion in gastric cancer, which in turn contributed to poor prognosis [30]. In our study, besides PRDX-5, residual disease but not age, clinical stage and tumor grade was an independent marker of poor prognosis. Byun et al., found that high expression of PRDX-5 was more frequently observed in advanced-stage endometrial cancer and tended to be associated with the presence of lymph node metastases but that the 5-year survival rate did not significantly differ depending on the PRDX-5 expression [31]. Similarly, in the study of Karihtala et al., an elevated cytoplasmic expression of PRDX-5 was associated with a higher stage of ovarian cancer but no analysis of survival was conducted [32].

Notably, in our study PRDX-5 expression was a negative prognostic indicator of survival both, in the entire study population and in the subgroup of $>50$-year-olds. However, further studies are needed as this finding may start the debate on different roles of PRDXs in hereditary and sporadic ovarian cancers since the population of patients aged $>50$ comprises fewer cases of hereditary ovarian cancer as compared to the younger group [33].

To the best of our knowledge, there has been only one study in patients with adrenocortical carcinoma which presented distinct results [34]. PRDX-5 expressed by cDNA microarray was 2.5-fold downregulated in adrenocortical carcinoma compared to benign adrenocortical tumors. These findings encouraged the authors to call PRX-5 a candidate 

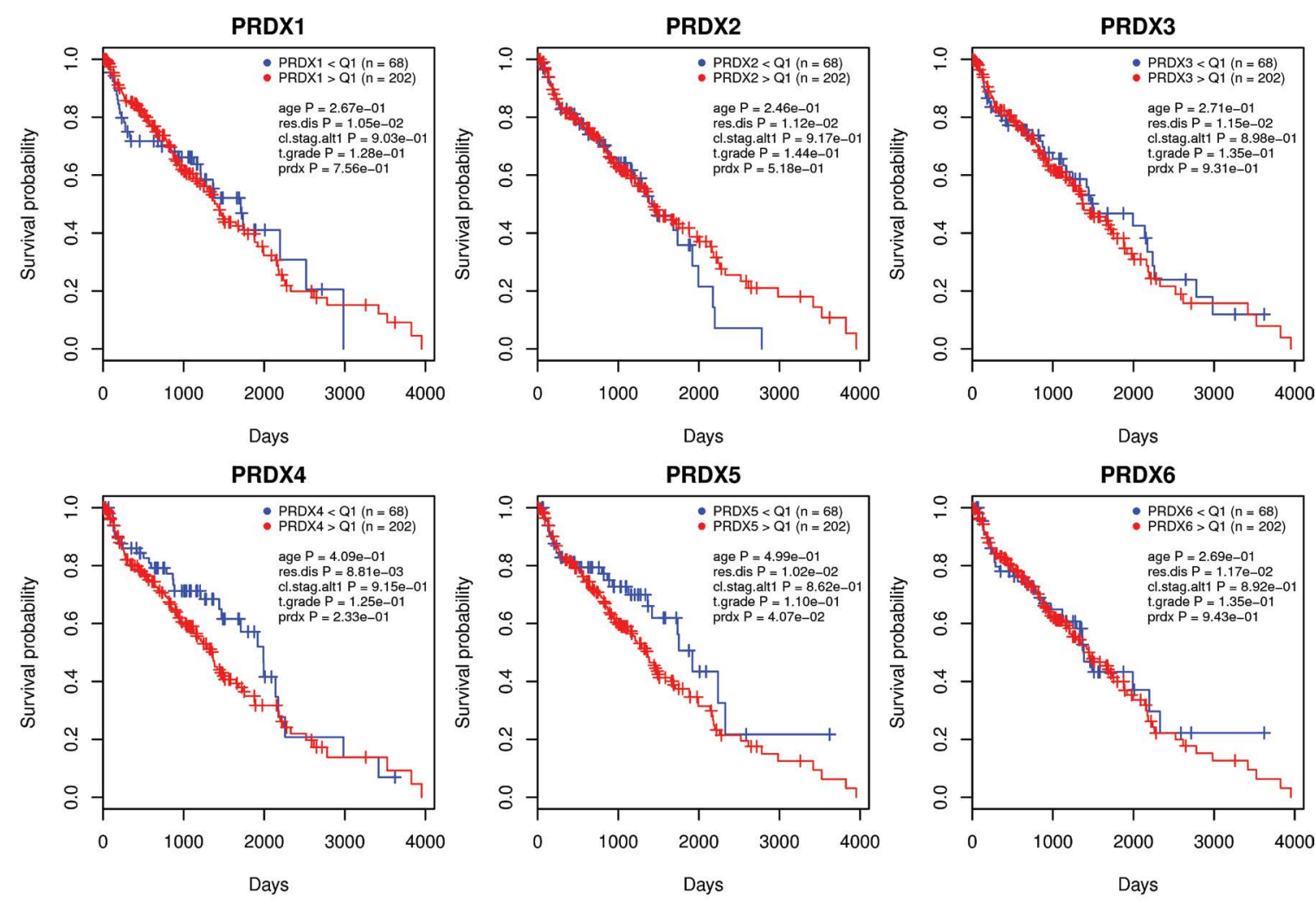

Figure 1. Kaplan-Meier analysis of the overall survival probability in all patients split based on the Q1 (1 ${ }^{\text {st }}$ quartile) threshold of PRDX 1-6 mRNA. Cox proportional hazard model regression was used to compute the significance of the clinical variables as well as the respective PRDX mRNA levels
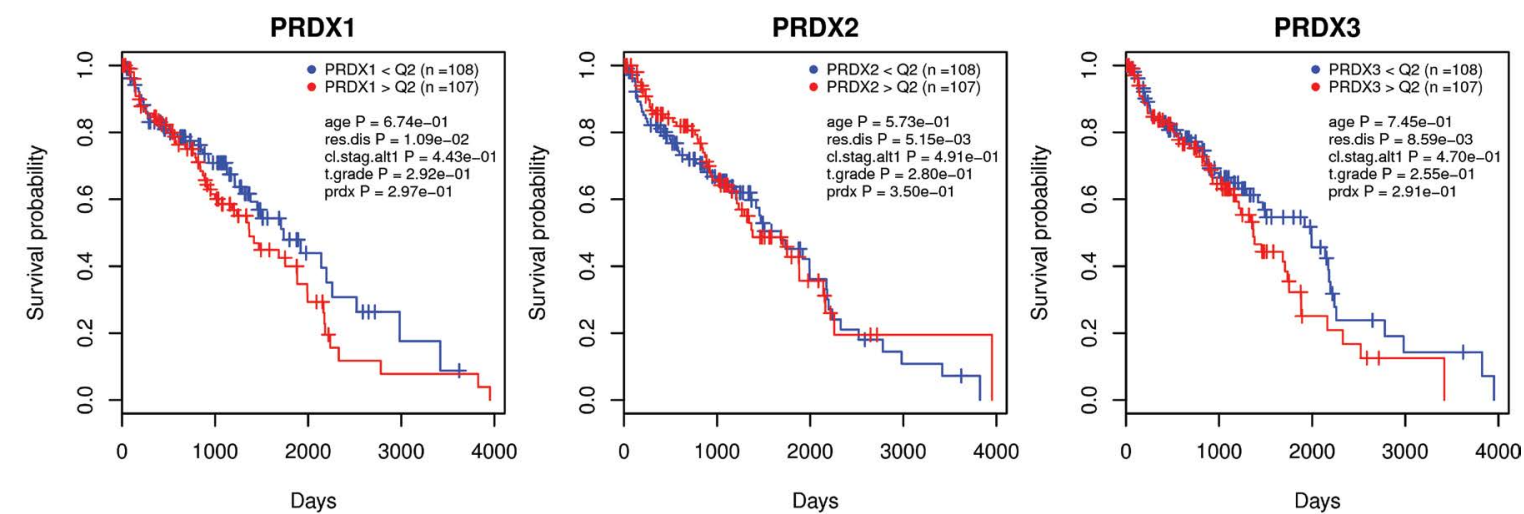

PRDX4
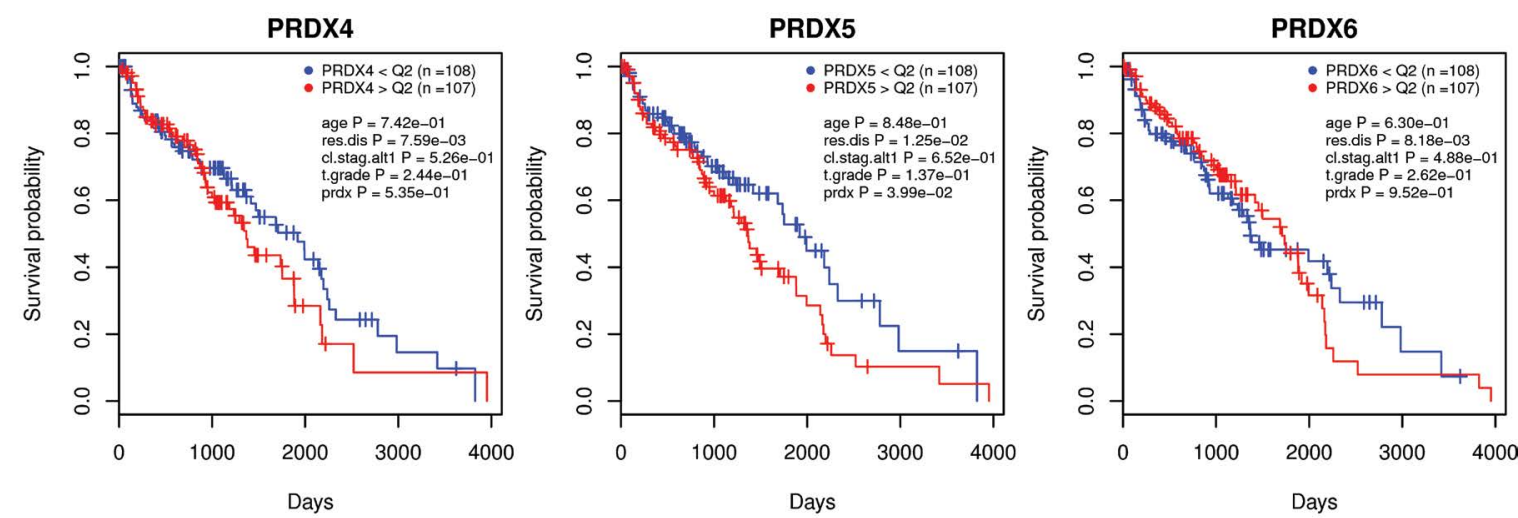

Figure 2. Kaplan-Meier analysis of the overall survival probability in patients over $50 \mathrm{y} / \mathrm{o}$ split based on the Q2 (median) threshold of PRDX 1-6 mRNA. Cox proportional hazard model regression was used to compute the significance of the clinical variables as well as the respective PRDX mRNA levels 
for the tumor suppressor gene and a diagnostic marker for distinguishing adrenocortical carcinoma from adenoma. However, no data on survival were available.

In this study, we found no association between PRDX1, 2, 3, 4 and 6 expression and patient survival. The number of studies investigating the association of PRDXs expression and survival in ovarian cancer is limited. Chung et al., compared PRDX-1 expression between serous borderline tumors and serous carcinoma and observed that PRDX-1 was overexpressed in most malignant ovarian tumors and correlated with poor overall survival in patients with ovarian serous carcinoma [35]. A study of Karihtala et al., evaluating 68 invasive ovarian carcinomas using immunohistochemistry reported that high cytoplasmic PRDX-4 immunostaining was associated with better prognosis [32].

Other reports showed an overexpression of PRDXs in OC tumors but the conclusions about the role of PRDXs in tumor progression are usually inconclusive or inconsistent. Li et al., based their opinion about the suppressive role of PRDX-2 in ovarian cancer progression only on its decreased expression in cancerous tissues compared with normal ovary [36]. The studies of PRDX-3 identified the subgroups of patients with a higher expression of this protein (poorly-differentiated, higher clinical stage) but found no relationship with OS or PFS $[37,38]$. In the abovementioned study of Karihtala et al., an elevated cytoplasmic expression rate of PRDX-6 was associated with a higher stage of $O C$ but no prognostic value of that protein was found [32]. To sum up, the available studies have demonstrated that PRDX-s overexpression is commonly observed in ovarian cancer, but the clinical significance of these findings remains to be elucidated.

Our study was not without limitations, chief among them the fact that the analysis was conducted on the mRNA level. We realize that the results should be repeated on the protein level. However, the dataset, although medium size, is relatively homogenic as it comes from one database, which strengthens the evidence.

\section{CONCLUSIONS}

PRDX-5 is a negative marker of survival in ovarian cancer patients and further studies are necessary to learn more about the specific role of PRDX-5 in ovarian cancer.

\section{Acknowledgments}

The work was supported by the Ministry of Science and Higher Education grant (IP2012 048172; DN).

\section{REFERENCES:}

1. Siegel RL, Miller KD, Jemal A. Cancer statistics, 2018. CA Cancer J Clin. 2018; 68(1): 7-30, doi: 10.3322/caac.21442, indexed in Pubmed: 29313949.

2. Henderson JT, Webber EM, Sawaya GF. Screening for Ovarian Cancer: Updated Evidence Report and Systematic Review for the US Preventive Services Task Force. JAMA. 2018; 319(6): 595-606, doi: 10.1001/jama.2017.21421, indexed in Pubmed: 29450530.

3. Coleman MP, Forman D, Bryant $\mathrm{H}$, et al. ICBP Module 1 Working Group Cancer survival in Australia, Canada, Denmark, Norway, Sweden, and the UK, 1995-2007 (the International Cancer Benchmarking Partnership): an analysis of population-based cancer registry data. Lancet. 2011; 377(9760): 127-138, doi: 10.1016/S0140-6736(10)62231-3, indexed in Pubmed: 21183212

4. Balmaña J, Tung NM, Isakoff SJ, et al. Phase I trial of olaparib in combination with cisplatin for the treatment of patients with advanced breast, ovarian and other solid tumors. Ann Oncol. 2014; 25(8): 1656-1663, doi: 10.1093/annonc/mdu187, indexed in Pubmed: 24827126.

5. Wood ZA, Schröder E, Robin Harris J, et al. Structure, mechanism and regulation of peroxiredoxins. Trends Biochem Sci. 2003; 28(1): 32-40, indexed in Pubmed: 12517450.

6. Jin DY, Chae HZ, Rhee SG, et al. Regulatory role for a novel human thioredoxin peroxidase in NF-kappaB activation. J Biol Chem. 1997; 272(49): 30952-30961, indexed in Pubmed: 9388242.

7. Nicolussi A, D'Inzeo S, Capalbo C, et al. The role of peroxiredoxins in cancer.MolClin Oncol.2017;6(2):139-153, doi: 10.3892/mco.2017.1129, indexed in Pubmed: 28357082.

8. Park MiH, Jo M, Kim YuRi, et al. Roles of peroxiredoxins in cancer, neurodegenerative diseases and inflammatory diseases. Pharmacol Ther. 2016; 163: 1-23, doi: 10.1016/j.pharmthera.2016.03.018, indexed in Pubmed: 27130805.

9. O'Leary PC, Terrile M, Bajor M, et al. Peroxiredoxin-1 protects estrogen receptor a from oxidative stress-induced suppression and is a protein biomarker of favorable prognosis in breast cancer. Breast Cancer Res. 2014; 16(4): R79, doi: 10.1186/bcr3691, indexed in Pubmed: 25011585.

10. Lee DJ, Kang DH, Choi $M$, et al. Peroxiredoxin-2 represses melanoma metastasis by increasing $\mathrm{E}$-Cadherin/ $\beta$-Catenin complexes in adherens junctions. Cancer Res. 2013; 73(15): 4744-4757, doi: 10.1158/0008-5472. CAN-12-4226, indexed in Pubmed: 23749642.

11. Choi JH, Kim TN, Kim S, et al. Overexpression of mitochondrial thioredoxin reductase and peroxiredoxin III in hepatocellular carcinomas. Anticancer Res. 2002; 22(6A): 3331-3335, indexed in Pubmed: 12530083.

12. Karihtala $P$, Mäntyniemi $A$, Kang SW, et al. Peroxiredoxins in breast carcinoma. Clin Cancer Res. 2003; 9(9):3418-3424, indexed in Pubmed: 12960131.

13. Kim YS, Lee HL, Lee KiB, et al. Nuclear factor E2-related factor 2 dependent overexpression of sulfiredoxin and peroxiredoxin III in human lung cancer. Korean J Intern Med. 2011; 26(3): 304-313, doi: 10.3904/kjim.2011.26.3.304, indexed in Pubmed: 22016591.

14. Ummanni R, Barreto F, Venz S, et al. Peroxiredoxins 3 and 4 are overexpressed in prostate cancer tissue and affect the proliferation of prostate cancer cells in vitro. J Proteome Res. 2012; 11(4): 2452-2466, doi: 10.1021/pr201172n, indexed in Pubmed: 22424448.

15. Hu JX, Gao Q, Li L. Peroxiredoxin 3 is a novel marker for cell proliferation in cervical cancer. Biomed Rep. 2013; 1(2): 228-230, doi: 10.3892/br.2012.43, indexed in Pubmed: 24648924.

16. Pritchard C, Mecham B, Dumpit R, et al. Conserved gene expression programs integrate mammalian prostate development and tumorigenesis. Cancer Res. 2009; 69(5): 1739-1747, doi: 10.1158/0008-5472. CAN-07-6817, indexed in Pubmed: 19223557.

17. Chang KP, Yu JS, Chien KY, et al. Identification of PRDX4 and P4HA2 as metastasis-associated proteins in oral cavity squamous cell carcinoma by comparative tissue proteomics of microdissected specimens using iTRAQ technology. J Proteome Res. 2011; 10(11): 4935-4947, doi: 10.1021/pr200311p, indexed in Pubmed: 21859152.

18. Karihtala P, Kauppila S, Soini $Y$, et al. Oxidative stress and counteracting mechanisms in hormone receptor positive, triple-negative and basal-like breast carcinomas. BMC Cancer. 2011; 11: 262, doi: 10.1186/1471-240711-262, indexed in Pubmed: 21693047.

19. Jiang $\mathrm{H}, \mathrm{Wu} \mathrm{L}, \mathrm{Mishra} \mathrm{M}$, et al. Expression of peroxiredoxin 1 and 4 promotes human lung cancer malignancy. Am J Cancer Res. 2014; 4(5): 445-460, indexed in Pubmed: 25232487.

20. Bur H, Haapasaari KM, Turpeenniemi-Hujanen $\mathrm{T}$, et al. Oxidative stress markers and mitochondrial antioxidant enzyme expression are increased in aggressive Hodgkin lymphomas. Histopathology. 2014; 65(3): 319-327, doi: 10.1111/his.12389, indexed in Pubmed: 24698430.

21. Kinnula VL, Lehtonen $S$, Sormunen $R$, et al. Overexpression of peroxiredoxins I, II, III, V, and VI in malignant mesothelioma. J Pathol. 2002; 196(3): 316-323, doi: 10.1002/path.1042, indexed in Pubmed: 11857495.

22. Karihtala P, Mäntyniemi A, Kang SW, et al. Peroxiredoxins in breast carcinoma. Clin Cancer Res. 2003; 9(9):3418-3424, indexed in Pubmed: 12960131. 
23. Pylväs $M$, Puistola $U$, Kauppila $S$, et al. Oxidative stress-induced antioxidant enzyme expression is an early phenomenon in ovarian carcinogenesis. Eur J Cancer. 2010; 46(9): 1661-1667, doi: 10.1016/j. ejca.2010.02.006, indexed in Pubmed: 20206498.

24. Gérard AC, Many MC, Daumerie Ch, et al. Peroxiredoxin 5 expression in the human thyroid gland. Thyroid. 2005; 15(3): 205-209, doi: 10.1089/thy.2005.15.205, indexed in Pubmed: 15785239.

25. Chang XZ, Li DQ, Hou YF, et al. Identification of the functional role of peroxiredoxin 6 in the progression of breast cancer. Breast Cancer Res. 2007; 9(6): R76, doi: 10.1186/bcr1789, indexed in Pubmed: 17980029.

26. Raatikainen S, Aaaltomaa S, Kärjä V, et al. Increased Peroxiredoxin 6 Expression Predicts Biochemical Recurrence in Prostate Cancer Patients After Radical Prostatectomy. Anticancer Res. 2015; 35(12): 6465-6470, indexed in Pubmed: 26637857.

27. Zhu H, Santo A, Li Y. The antioxidant enzyme peroxiredoxin and its protective role in neurological disorders. Exp Biol Med (Maywood). 2012; 237(2): 143-149, doi: 10.1258/ebm.2011.011152, indexed in Pubmed: 22302711.

28. Nguyên-Nhu NT, Berck J, Clippe A, et al. Human peroxiredoxin 5 gene organization, initial characterization of its promoter and identification of alternative forms of mRNA. Biochim Biophys Acta. 2007; 1769(7-8): 472483, doi: 10.1016/j.bbaexp.2007.05.004, indexed in Pubmed: 17628720.

29. Graves JA, Metukuri M, Scott D, et al. Regulation of reactive oxygen species homeostasis by peroxiredoxins and c-Myc. J Biol Chem. 2009; 284(10):6520-6529, doi: 10.1074/jbc.M807564200, indexed in Pubmed: 19098005.

30. Kim B, Kim YS, Ahn HM, et al. Peroxiredoxin 5 overexpression enhances tumorigenicity and correlates with poor prognosis in gastric cancer. Int J Oncol. 2017; 51(1): 298-306, doi: 10.3892/ijo.2017.4013, indexed in Pubmed: 28535004.

31. Byun JMi, Kim SuS, Kim KiT, et al. Overexpression of peroxiredoxin-3 and -5 is a potential biomarker for prognosis in endometrial cancer. Oncol Lett. 2018; 15(4): 5111-5118, doi: 10.3892/ol.2018.7909, indexed in Pubmed: 29541251.
32. Karihtala P, Soini Y, Vaskivuo L, et al. DNA adduct 8-hydroxydeoxyguanosine, a novel putative marker of prognostic significance in ovarian carcinoma. Int J Gynecol Cancer. 2009; 19(6): 1047-1051, doi: 10.1111/IGC.0b013e3181ad0f0d, indexed in Pubmed: 19820366.

33. Kotsopoulos J, Gronwald J, Karlan B, et al. Hereditary Ovarian Cancer Clinical Study Group, Hereditary Breast Cancer Clinical Study Group, Hereditary Breast Cancer Clinical Study Group, Hereditary Breast Cancer Clinical Study Group, Hereditary Breast Cancer Clinical Study Group, Hereditary Breast Cancer Clinical Study Group, Hereditary Breast Cancer Clinical Study Group, Hereditary Breast Cancer Clinical Study Group, Hereditary Breast Cancer Clinical Study Group. Timing of oral contraceptive use and the risk of breast cancer in BRCA1 mutation carriers. Breast Cancer Res Treat. 2014; 143(3): 579-586, doi: 10.1007/s10549-013-2823-4, indexed in Pubmed: 24458845.

34. Fernandez-Ranvier GG, Weng J, Yeh RF, et al. Candidate diagnostic markers and tumor suppressor genes for adrenocortical carcinoma by expression profile of genes on chromosome 11q13. World J Surg. 2008; 32(5): 873-881, doi: 10.1007/s00268-008-9521-0, indexed in Pubmed: 18324346.

35. Chung KH, Lee DH, Kim Y, et al. Proteomic identification of overexpressed PRDX 1 and its clinical implications in ovarian carcinoma. J Proteome Res. 2010; 9(1): 451-457, doi: 10.1021/pr900811x, indexed in Pubmed: 19902980.

36. Li XQ Zhang SL, Cai Z, et al. Proteomic identification of tumor-associated protein in ovarian serous cystadenocarinoma. Cancer Lett. 2009; 275(1): 109-116, doi: 10.1016/j.canlet.2008.10.019, indexed in Pubmed: 19056166.

37. Duan J, Lang Y, Song C, et al. siRNA targeting of PRDX3 enhances cisplatininduced apoptosis in ovarian cancer cells through the suppression of the NFKB signaling pathway. Mol Med Rep. 2013; 7(5): 1688-1694, doi: 10.3892/mmr.2013.1370, indexed in Pubmed: 23503975.

38. Wang Xy, Wang $\mathrm{Hj}$, Li Xq. Peroxiredoxin III protein expression is associated with platinum resistance in epithelial ovarian cancer. Tumour Biol. 2013; 34(4): 2275-2281, doi: 10.1007/s13277-013-0769-0, indexed in Pubmed: 23564483. 